\section{Labour analgesia in a parturient with lumbar tattoo: a routine management? or not?}

To the Editor:

Douglas and Swenerton first reported on the administration of labour analgesia in three parturients with lumbar tattoos and discussed the possible ramifications of neuraxial analgesia in women with tattoos in the lumbar area. ${ }^{1}$ All three women received uneventful epidural analgesia, and no anesthesia-related complications were reported. I herein present a similar case, and the first report of a minor anesthesia complication resulting from epidural needle insertion through tattoos during the performance of neuraxial block. A 34-yr-old, healthy female at term was in labour and requested labour analgesia. Preanesthetic back examination revealed the colourful tattoos covering her entire lumbar area. An epidural block was performed in a standard manner (one attempt at L2-3 interspace) with an 18-gauge Tuohy needle. Several hours after an uneventful delivery, the patient reported tenderness and burning in the lumbar area where the epidural catheter had been sited. Examination revealed a localized L2 -3 interspace tenderness, however, due to the presence of a tattoo in this area, skin redness (irritation) could not be determined. The neurological examination was normal and her symptoms resolved over the next $24 \mathrm{hr}$. A pigment-containing tissue core from a tattoo seems a possible cause of deeper lumbar tissue irritation.

Krzysztof M. Kuczkowski MD

San Diego, California

\section{Reference}

I Douglas MJ, Swenerton JE. Epidural anesthesia in three parturients with lumbar tattoos: a review of possible implications. Can J Anesth 2002; 49: 1057-60.

\section{REPLY:}

Thank you for the opportunity of commenting on this case of pain following insertion of an epidural needle through a tattoo. Our article dealt with the theoretical risk of a core of tissue containing pigment from a tattoo causing long-term neurological problems. ${ }^{l}$ Dr. Kuczkowski describes tenderness and burning at the site of the epidural catheter several hours after it was sited through a lumbar tattoo. Fortunately, the symptoms resolved over $24 \mathrm{hr}$ but the author wonders whether the symptoms were related to passage of the epidural needle through the tattoo with resulting irritation from the tattoo pigment.
Although difficult to say with certainty, a more likely explanation is that the tenderness was related to bruising from the epidural needle, an occurrence that is seen in women without tattoos. However, as more individuals are being tattooed complications related to neuraxial blocks done through the tattoo may be reported.

\section{Joanne Douglas MD FRCPC}

Jean E. Swenerton MD FRCPC

Vancouver, British Columbia

\section{Reference}

1 Douglas MJ, Swenerton JE. Epidural anesthesia in three parturients with lumbar tattoos: a review of possible implications. Can J Anesth 2002; 49: 1057-60.

\section{Altered bupivacaine pharmacokinetics by $\mathrm{MgSO}_{4}$ in rats}

To the Editor:

We previously reported that magnesium sulfate $\left(\mathrm{MgSO}_{4}\right)$ increased the threshold of bupivacaineinduced seizures in rats. ${ }^{1}$ Since it is not clear that the pharmacokinetic interaction of bupivacaine with concomitant $\mathrm{MgSO}_{4}$ involves the mechanism of the inhibitory effect of $\mathrm{MgSO}_{4}$ on seizure, we planned the following study, using awake rats.

Eleven Sprague-Dawley rats were chronically catheterized and divided into two groups; five in Group $\mathrm{M}$ received an $i v$ bolus dose $\left(50 \mathrm{mg} \cdot \mathrm{kg}^{-1}\right)$ of $\mathrm{MgSO}_{4}$ over two minutes, followed by an infusion of 4 $\mathrm{mg} \cdot \mathrm{kg}^{-1} \cdot \mathrm{min}^{-1}$ throughout the experiment. Six in Group $S$ received an equal volume of normal saline. After two hours of the pretreatment with either solution, a bupivacaine bolus of $1 \mathrm{mg} \cdot \mathrm{kg}^{-1}$ was administered intravenously, followed by an infusion of $0.4 \mathrm{mg} \cdot \mathrm{kg}^{-1} \cdot \mathrm{min}^{-1}$ for $15 \mathrm{~min}$. Serial arterial samples were obtained after the bupivacaine infusion to measure the concentrations of magnesium, bupivacaine and its major metabolite, 3'-hydroxybupivacaine (3'-OH-Bup). We also measured those concentrations in the liver at the end of the study. The concentration-time profile during and following the bupivacaine administration was fitted to a two-compartment model, using ADAPT II. ${ }^{2}$ The estimated intercepts and slopes were used for the calculation of standard pharmacokinetic variables. Comparison was performed separately, using a t test $(P<0.05)$.

Serum magnesium concentrations and the pharmacokinetic variables are shown in the Table (available as additional material at www.cja-jca.org). In three rats of Group M, 3'-OH-Bup was detected over 60 min after 